\title{
NOTE ON LEGENDRE NUMBERS
}

\section{R. SITARAMACHANDRARAO}

Dept. of Mathematics

Andhra University

Walfair 53003 India

\section{B.R. DAVIS}

Dept. of Mathematics University of Toledo Toledo, Ohio 43606

(Received May 18, 1987)

ABSTRACT. The definition and basic properties of Legendre Numbers are reviewed here. We then develop some new properties and identities involving sums of Legendre Numbers, including clarification of a statement in the paper of Haggard [1].

KEY WORDS AND PHRASES. Legendre Numbers, Stirling's formula, sums of reciprocals of of Legendre Numbers, Abel sum.

1980 AMS SUBJECT CLASSIFICATION CODE. 10A40, $26 \mathrm{C} 99$.

\section{INTRODUCTION}

Recently P. W. Haggard [1] introduced Legendre Numbers, discussed various of their properties, and evaluated certain related infinite series and integrals. In this note we review some of these ideas and discuss some further results.

1. LEGENDRE NUMBERS.

The Legendre polynomials $P_{n}(x)$ are defined [2] by the generating function

$$
\left(1-2 x t+t^{2}\right)^{-1 / 2}=\sum_{n=0}^{\infty} P_{n}(x) t^{n}
$$

and the Associated Legendre functions are defined by

$$
P_{n}^{m}(x)=\left(1-x^{2}\right)^{\frac{m}{2}} D^{m}\left(p_{n}(x)\right) \text {. }
$$

Recently P. W. Haggard [1] defined the Legendre Number, $P_{n}^{m}$, to be $P_{n}^{m}(0)$ and studied some of their basic properties. By the well-known Rodrigue's formula [2]

$$
P_{n}(x)=\frac{1}{2^{n} \cdot n !} D^{n}\left(\left(x^{2}-1\right)^{n}\right),
$$

we see that

$$
P_{n}^{m}(x)=\frac{\left(1-x^{2}\right)^{\frac{m}{2}}}{2^{n} \cdot n !} D^{m+n}\left(\left(x^{2}-1\right)^{n}\right)
$$

and consequently

$$
P_{n}^{m}=P_{n}^{(m)}(0),
$$

where $P_{n}^{(m)}(0)$ is the value of the mth derivative of $P_{n}(x)$ at $x=0$. Haggard [1] deduced the following explicit formula from (1.4). 


$$
P_{n}^{m}=\left[\begin{array}{cl}
0 & m+n \text { odd } \\
0 & m>n \text { and } \\
\frac{(-1)^{\frac{n-m}{2}}(n+m) !}{2^{n}\left(\frac{n+m}{2}\right) !\left(\frac{n-m}{2}\right) !} & m+n \text { even, } m \leq n .
\end{array}\right.
$$

He also gave a table of $p_{n}^{m}$ for $0 \leq m, n \leq 8$.

We note that (1.6) follows directly from (1.1). In fact by (1.1) and the Binomial theorem

$$
\begin{aligned}
\sum_{n=0}^{\infty} P_{n}(x) t^{n} & =\{1-t(2 x-t)\}^{-1 / 2}=1+\sum_{n=1}^{\infty} \frac{1 \cdot 3 \cdot 5 \cdots(2 n-1)}{2^{n} \cdot n !} t^{n}(2 x-t)^{n} \\
& =1+\sum_{n=1}^{\infty} \frac{(2 n) !}{2^{2 n}(n !)^{2}} \sum_{k=0}^{n}(-1)^{k}\left(\begin{array}{l}
n \\
k
\end{array}\right)(2 x)^{n-k} t^{n+k} \\
& =1+\sum_{m=1}^{\infty} t^{m} \quad \sum_{\frac{m}{2} \leq n \leq m} \quad \frac{(-1)^{m-n}(2 n) !}{2}\left(\begin{array}{c}
n \\
m-n
\end{array}\right) x^{2 n-m}
\end{aligned}
$$

so that

$$
P_{n}(x)=2^{-n} \sum_{i=0}^{n / 2}(-1)^{i}\left(\begin{array}{c}
n-1 \\
i
\end{array}\right)\left(\begin{array}{c}
2(n-1) \\
n-1
\end{array}\right) x^{n-2 i}
$$

Writing $m$ for $n-2 i$ in this, we get

$$
P_{n}(x)=2^{-n} \sum_{\substack{m=0 \\
m+n \text { even }}}^{n}(-1)^{-\frac{n-m}{2}}\left(\begin{array}{l}
(n+m) / 2 \\
(n-m) / 2
\end{array}\right)\left(\begin{array}{l}
n+m \\
(n+m) / 2
\end{array}\right) x^{m} .
$$

Now since $P_{n}(x)=\sum_{m=0}^{n} \frac{P_{n}^{(m)}(0)}{m !} x^{m}$, we get $(1.6)$.

2. INTEGER VALUES OF $P_{n}^{m}$

In this section we prove that for $P_{n}^{m} \neq 0$, then $P_{n}^{m}$ is an integer iff $m=n$. For this, let $[x]$ denote the largest integer $\leq x$, and for prime $p$ and $n \geq 1$, let $H(p, n)$ denote the highest power of $p$ dividing $n$. Then it is well known, due to Legendre (cf. [3], p. 67), that

$$
H(p, n !)=\sum_{r=1}^{i}\left[\begin{array}{c}
n \\
p
\end{array}\right],
$$

THEOREM 2.1 The highest power of 2 dividing the denominator of $P_{n}^{n-2 k}$, $n \geq 1$ and $k \geq 0$, when expressed in $i$ ts lowest terms, is $k+H(2, k !)$. In particular, a non-zero Legendre number $P_{n}^{m}$ is an integer iff $m=n$.

PROOF. By 1.6 and 2.1 , letting $m=n-2 k$, we see that the highest power of 2 dividing the denominator of $P_{n}^{n-2 k}$ (in its reduced form) is given by 


$$
\begin{aligned}
H\left(2,2^{n}\left(n+\frac{n-2 k}{2}\right) !\left(\frac{n-n+2 k}{2}\right) !\right) \\
\quad=n+\sum_{r=1}^{\infty}\left[\frac{n+(n-2 k)}{2^{r}+1}\right]+\sum_{r=1}^{\infty}\left[\frac{n-(n-2 k)}{2^{r+1}}\right]-\sum_{r=1}^{\infty}\left[\frac{n+(n-2 k)}{2^{r}}\right] \\
\quad=n+\sum_{r=1}^{\infty}\left[\frac{n-k}{2^{r}}\right]-\sum_{r=0}^{\infty}\left[\frac{n-k}{2^{r}}\right]+\sum_{r=1}^{\infty}\left[\frac{k}{2^{r}}\right] \\
=n-(n-k)+\sum_{r=1}^{\infty}\left[\frac{k}{2^{r}}\right] \\
=k+H(2, k !) .
\end{aligned}
$$

Hence, if $m=n$, then $k=0$, and the highest power of 2 dividing the denominator of $P_{n}^{n}$ is zero and $P_{n}^{n}$ is an integer. If $k=0$, then $m=n$.

3. SUMS INVOLVING $P_{n}^{m}$.

Haggard [1] proved that

$$
\sum_{n=0}^{\infty} P_{n}^{0}=2^{-1 / 2}
$$

and for $k \geq 1$

$$
\sum_{n=k}^{\infty} p_{n}^{k}=1 \cdot 3 \cdot 5 \cdot 7 \cdots(2 k-1) 2^{-\left(\frac{2 k+1}{2}\right)} .
$$

However, we note that his arguments prove only that the stated sums in (3.1) and (3.2) are in the sense of Abel. In fact, as we show later, the series

$$
\sum_{n=k}^{\infty} p_{n}^{k} \text {, for fixed } k \geq 0 \text {, converges iff } k=0 \text {. }
$$

To see this, using Stirling's formula, viz.

we have

$$
n ! \sim \sqrt{2 \pi} n^{n+1 / 2} e^{-n} \text {, as } n \rightarrow \infty
$$

$$
\text { and hence } \begin{aligned}
{ }_{n}^{2 n} & \sim 2^{2 n}\left(n_{\pi}\right)^{-1 / 2}, \\
& P_{2 n}^{0}=(-1)^{n}\left(\begin{array}{c}
2 n \\
n
\end{array}\right) 2^{-2 n} \sim \frac{(-1)^{n}}{\sqrt{n \pi}}
\end{aligned}
$$

Also, since the sequence $\left\{\left|P_{2 n}^{0}\right|\right\}_{n}=0,1,2, \ldots$ is decreasing, the series $\sum_{n=0}^{\infty} p_{2 n}^{0}$ converges.

Now let $k \geq 1$ be fixed. Again by Stirling's formula, we see that

$$
p_{2 n+k}^{k}=(-1)^{n} \frac{(2 n+2 k) !}{n !(n+k) ! 2^{2 n+k}} \sim(-1)^{n} n^{k-1 / 2} 2^{k}-1 / 2
$$

and hence the series $\sum_{n=0}^{\infty} p_{2 n+k}^{k}$, for fixed $k \geq 1$, actually diverges.

However, some interesting sums involving reciprocals of Legendre numbers yield the following results. 
THEOREM 3.? For $|x| \cdot 1$

$$
\sum_{n=1}^{\infty} \frac{(-1)^{n} x^{2 n}}{n p_{2 n}^{0}}=\frac{2 x \sin ^{-1} x}{\sqrt{1-x^{2}}}
$$

FROOF. it is known from Lehmer [4] that for $|x|<1$

$$
\sum_{n=i}^{\infty} \frac{(2 x)^{2 n}}{n\left(\begin{array}{c}
2 n \\
n
\end{array}\right)}=\frac{2 x \cdot \sin ^{-1} x}{\sqrt{1-x^{2}}}
$$

and (3.3) is a reformulation of this.

COROLLARY 3.1 For $|x|<1$

$$
\sum_{n=1}^{\infty} \frac{(-1)^{n}-x^{2 n}}{n^{2} p_{2 n}^{0}}=2\left(\sin ^{-1} x\right)^{2}
$$

by dividing (3.3) by $x$ and integrating both sides,

$$
\sum_{n=1}^{\infty} \frac{(-1)^{n} x^{2 n}}{p_{2 n}^{0}}=\frac{x^{2}}{1-x^{2}}+\frac{x \sin ^{-1} x}{\left(1-x^{2}\right)^{3 / 2}}
$$

by differentiation of (3.3) and multiplying by $x$, and then,

$$
\begin{aligned}
& \sum_{n=1}^{\infty} \frac{x^{2 n}}{n} p_{2 r_{0}}^{0}=-\frac{2 x\left(\sinh ^{-1} x\right)}{\sqrt{1+x^{2}}} \\
& \sum_{n=1}^{n}-\frac{x^{2} n}{n^{2} p_{2 n}^{0}}=-2\left(\sinh ^{-1} x\right)^{2} \\
& \sum_{n=1}^{m} \frac{x^{2 n}}{p_{2 n}^{0}}=-\left\{x^{2}+\frac{x \sinh ^{-1} x}{\left(1+x^{2}\right)^{3 / 2}}\right\}
\end{aligned}
$$

by replacing $x$ by $i x$ in $3.3,3.4$ and 3.5 .

REMARK 3.1 Since $P_{2 n-1}^{1}=-2 n P_{2 n}^{0}$, results corresponding to Theorem 3.1 and Corollary 3.1 can be formulated for sums involving $p_{2 n-1}$. For various special cases we refer the reacer to the very interesting paber of $D$. H. Lehmer [4]. However, it appears that obtaining a closed expresston for the sums of series such as

$\sum_{\substack{n=m \\ n+m}}^{\infty} \frac{x^{2 n}}{P_{n}^{m}}$, for larger $m$, is a difficult problem.

\section{REFERENCES}

1. HAGGARD, P.W. On Legendre Numbers, Internat. J. Math. and Math. Sci., $\underline{8}$ (1985), 407-411.

2. RAINVILLE, E.J. Soecial Functions, The Macmillan Company, Nek' York, 1960.

3. APOSTOL, T.M. Introduction to Analytic Number Theory, Undergraduate Tes ts in Mathematics, Springer-Verias, New York, 1975.

4. LEHMER, D. H. Interesting Series invoiving the Central Binomial Coefficient, The American Miathematical Monthly, Vol. 92 (1985), 449-457. 


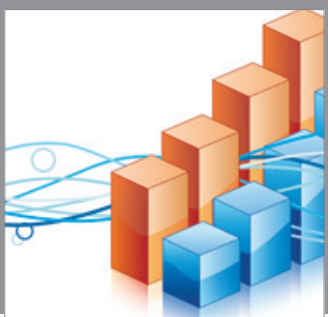

Advances in

Operations Research

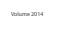

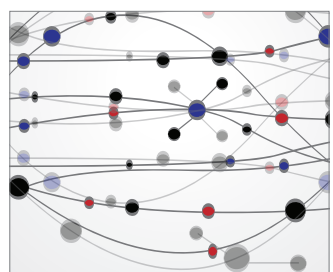

\section{The Scientific} World Journal
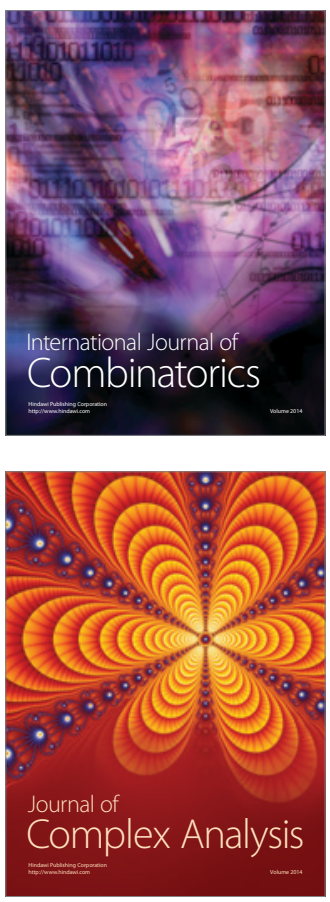

International Journal of

Mathematics and

Mathematical

Sciences
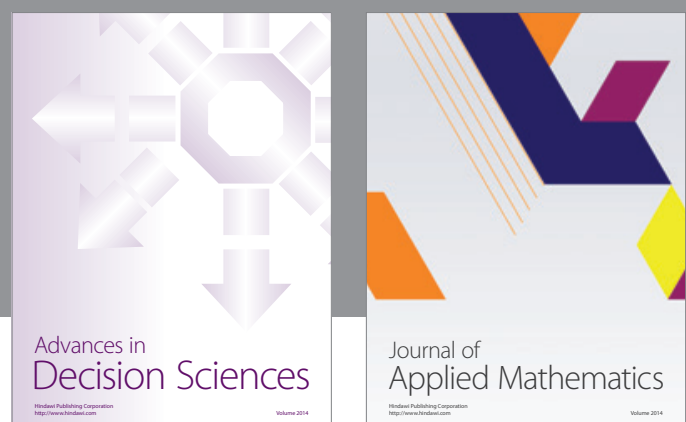

Journal of

Applied Mathematics
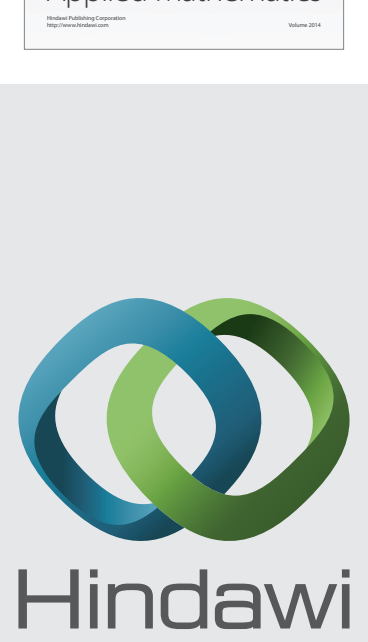

Submit your manuscripts at http://www.hindawi.com
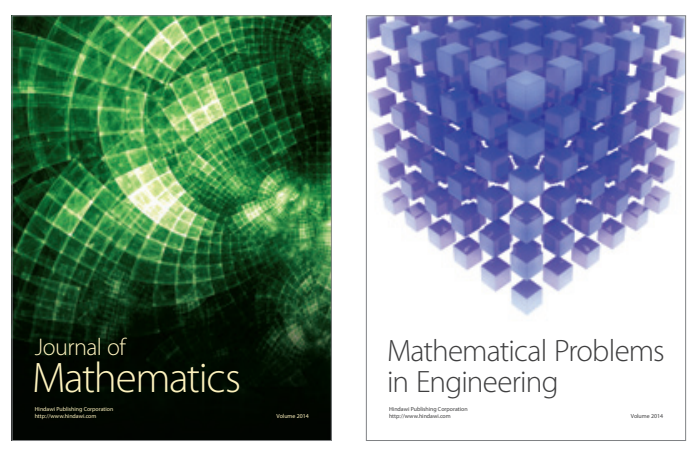

Mathematical Problems in Engineering
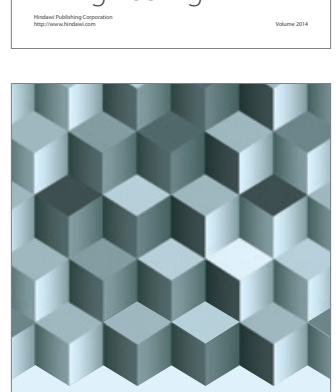

Journal of

Function Spaces
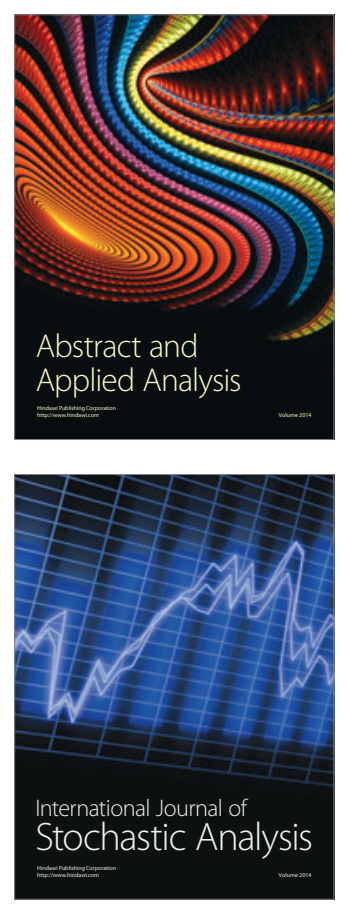

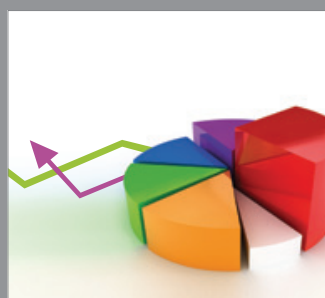

ournal of

Probability and Statistics

Promensencen
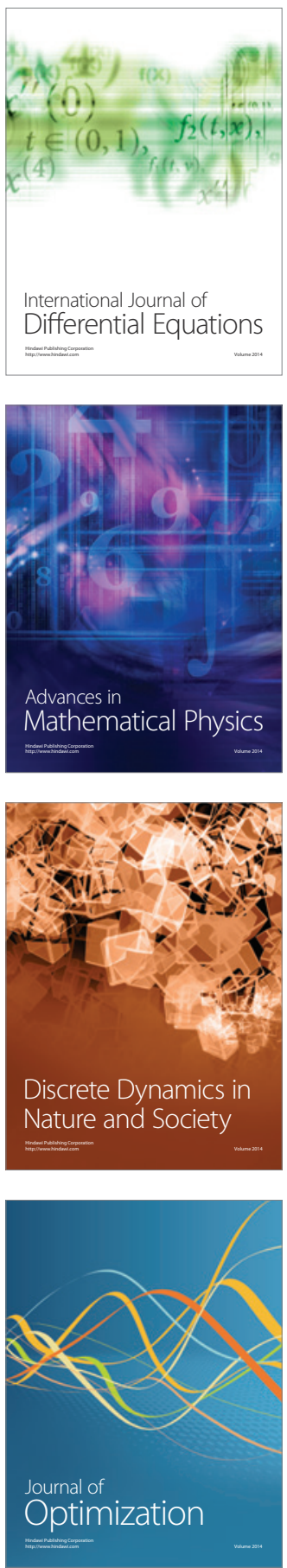